\title{
Accuracy of predictive factors for difficult intubation
}

\author{
Ying Gao ${ }^{1} \cdot$ Shao-Hua Liu ${ }^{1}$ Fu-Shan Xue ${ }^{1}$ \\ Received: 29 January 2020 / Accepted: 21 March 2020 / Published online: 27 March 2020 \\ (c) Japanese Society of Anesthesiologists 2020
}

Keywords Prediction $\cdot$ Risk factors $\cdot$ Difficult intubation

\section{To the Editor:}

In recent article of Mostafa et al. [1], we noted several issues that would have made interpretation of their findings difficult. First, limited neck extension (LNE) is a known risk factor for difficult airways. In multivariate analysis, odds ratios of LNE for difficult laryngoscopy and intubation were significantly greater than those of low thyromental height test and high modified Mallampati test, but LNE was not identified as a risk factor. In method, the authors did not clearly define the LNE and class its severity. Second, difficult laryngoscopy is a main determinant of successful intubation with direct laryngoscopy, but incidence of difficult laryngoscopy (12\%) in this study was lower than incidence of difficult intubation was $17 \%$. It was unclear what tracheal tubes were used and why first intubation trial was done without the use of a stylet. By providing increased rigidity and malleability, the use of a stylet allows more control of tube and facilitates successful intubation. Third, the authors calculated the AUROC, positive and negative predictive values, sensitivity, specificity and best cut-off values of all predictive parameters for difficult laryngoscopy and intubation, but did not provide their Youden indexes. Actually, Youden index is a direct measure of diagnostic accuracy at the best cutoff value, i.e., maximum overall correct classification rate that a predictive parameter can achieve [2]. In other words, even if a predictive parameter with a large AUROC may have an unsatisfactory overall correct classification rate at the best cutoff point, and vice versa.

Acknowledgements All authors have no financial support and potential conflicts of interest for this work.

\section{Compliance with ethical standards}

Conflict of interest None declared.

\section{References}

1. Mostafa M, Saeed M, Hasanin A, Badawy S, Khaled D. Accuracy of thyromental height test for predicting difficult intubation in elderly. J Anesth. 2019. https://doi.org/10.1007/s00540-01902721-6

2. Yin J, Tian L. Joint confidence region estimation for area under ROC curve and Youden index. Stat Med. 2014;33:985-1000.

Publisher's Note Springer Nature remains neutral with regard to jurisdictional claims in published maps and institutional affiliations.

This comment refers to the article available at https://doi. org/10.1007/s00540-019-02721-6.

\author{
Fu-Shan Xue \\ xuefushan@aliyun.com \\ 1 Department of Anesthesiology, Beijing Friendship \\ Hospital, Capital Medical University, No. 95 \\ Yong-An Road, Xi-Cheng District, Beijing 100050, \\ People's Republic of China
}

